\title{
Plasmonic Lipid Bilayer Membranes for Enhanced Detection Sensitivity of Biolabeling Fluorophores.
}

\author{
R. Bhattacharya*1,2, Chaitanya Indukuri ${ }^{2}$, N. Begam ${ }^{2}$, Oliver H. Seeck ${ }^{3}$ and J. K. Basu ${ }^{2}$ \\ ${ }^{1}$ Department of Physics, University of California- San Diego, 9500 Gilman Dr. La Jolla, CA, \\ 92093, USA. \\ ${ }^{2}$ Department of Physics, Indian Institute of Science, Bangalore, 560012, India. \\ ${ }^{3}$ Deutsches Elektronen-Synchrotron DESY, 22607 Hamburg, Germany.
}

September 13, 2015

\begin{abstract}
Plasmonics based sensing using the surface plasmon resonance of metal nanoparticles has been effectively demonstrated in various applications. Extending this methodology to cell and artificial lipid bilayer membranes would be extremely beneficial in enhancing the sensitivity of the detection of binding and cellular transport of molecules across such membranes. Here we demonstrate the creation of a artificial plasmonic biomembrane template and use it to demonstrate the enhanced detection sensitivity of certain widely used biomarker molecules. The efficacy of these templates are explained in terms of the ability of the hydrophobic polymer grafted gold nanoparticles, used, to organize, penetrate and fluidize the membranes. The enhancement of photoluminescence of the dye molecules used occurs over a reasonably large spectral range as compared to the plasmon resonance of gold nanoparticles. The results could, possibly, be extended to cellular membrane with relevant modifications, as well as to detection of any other biological molecules, appropriately labeled with fluorescent dye molecules and demonstrates the versatility of these plasmonic bio-inspired platforms as potential bio-chemical sensors.
\end{abstract}

*rupak@physics.ucsd.edu, basu@physics.iisc.ernet.in

\section{Introduction}

Binding of molecules to cellular or biomimetic membranes is an important process in functioning and signaling aspect of such membranes.Usually such binding events would trigger changes both on and within the membrane. Enhanced detection sensitivity to such binding could help correlate, better, the binding events to the subsequent structural, physical or chemical /biological process which occur as a consequence. One of the methods by which such sensitivity could be enhanced is by using metal nanoparticles(NP), especially gold( $\mathrm{Au}$ ) and silver(Ag), which by virtue of their ability to generate large local nanoscale electric field due to their surface plasmon resonance(SPR) [1, 2, 3, 4, 5] . However, while the use of such NP or their oligomers or arrays have been shown to be effective in enhanced sensitivity of molecules for instance, through surface enhanced Raman scattering(SERS) [6, 7, 8, 9, 10] or photoluminescence (PL) enhancement [11, 12, 13, 14], their use in bioinspired membranes have not been very fruitful.

Hydrophobicity of molecules is one of the crucial parameters which controls the extent of their membrane penetration. The interaction of various types of hydrophobic molecules and model lipid membrane have been studied extensively using both computer simulations $[15,16,17$, [18] and experiments [19, 20, 21, 22]. A wide variety of 


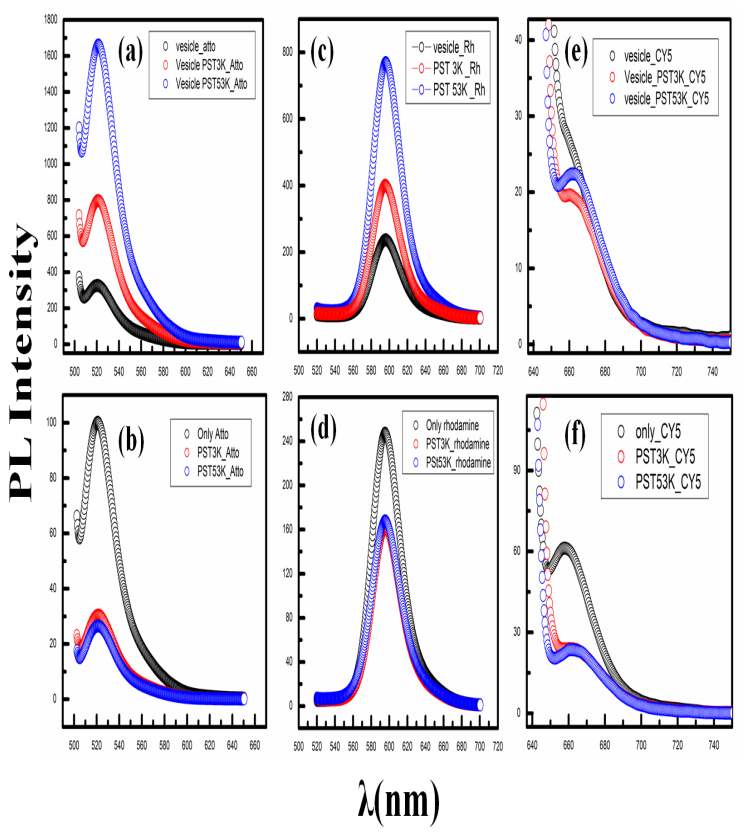

Figure 1 Bulk PL measurements of Atto488 dye in presence of PGNP incubated SUVs(a) and only PGNPS without vesicles(b). Similar measurements for rhodamine dye in presence of PGNPs with (c) and without SUVs(d). PL measurements for CY5 dye in presence of PGNPs with (e) and in without SUVs(f).

hydrophobic molecules including proteins, flexible polymers, branched dendrimers as well as hydrophobic soft nanoparticles or star polymers interact with model membranes and penetrate it either by changing their conformational change or disrupting the local morphology of the underlying membrane [19, 23, 24, 25, 18]. In addition to hydrophobicity, the size of the nanoparticle also matters for membrane absorption[21, 16, 26, 27, 17, 28, 29], especially with respect to the hydrophobic mis-match. Depending on their size, nanoparticles can deform and thus trigger a different morphological as well as mechanical transition [27, 29, 30] in model membranes.

Here, we use hydrophobic, polymer grafted AuNPs (PGNP) of suitable sizes, embedded in lipid bilayer membranes, to create plasmonic bio compatible membranes. Using these plasmonic membranes we demonstrate sig-
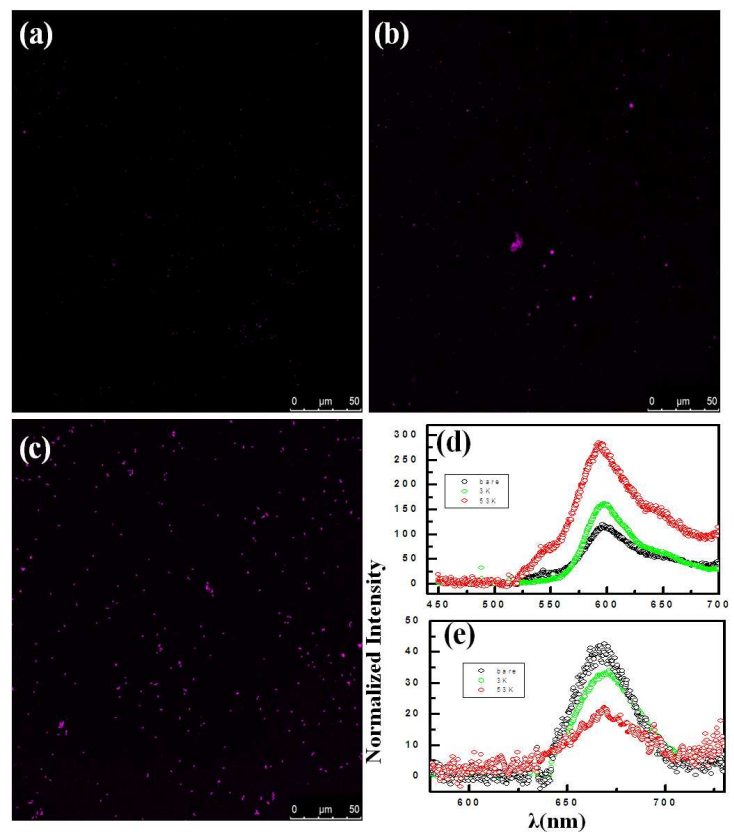

Figure 2 Confocal fluorescence images of $\mathrm{Rh}$ at concentration 870nM on (a) bare DMPC SLBs, (b) PST3K PGNP incubated DMPC SUVs, (c) PST53K PGNP incubated DMPC SLBs. Comparison of PL emission spectra for (d) Rh and (e) CY5 at same dye concentration (as in (a)-(c)) on DMPC SLBs with and without the presence of PST3K and PST53K PGNPs.

nificant enhancement of detection sensitivity of binding of prototypical molecules, Atto 488 and Rhodamine to such membranes. The modification of photoluminescence(PL) of these dye molecules have been demonstrated for both vesicles as well as supported lipid bilayer membranes(SLB). Detailed atomic force microscopy(AFM) as well as grazing incidence X-ray scattering (GIXS) measurements on supported lipid bilayer(SLB) membranes elucidate the mechanism underlying the observed PL intensity variation of these dye molecules in terms of self assembled pattern formation, penetration and membrane reorganization by the PGNPs. The results could be extended to cellular membrane with appropriate modification as well as to detection of any other biological molecules, appropriately labeled with fluorescent dye 

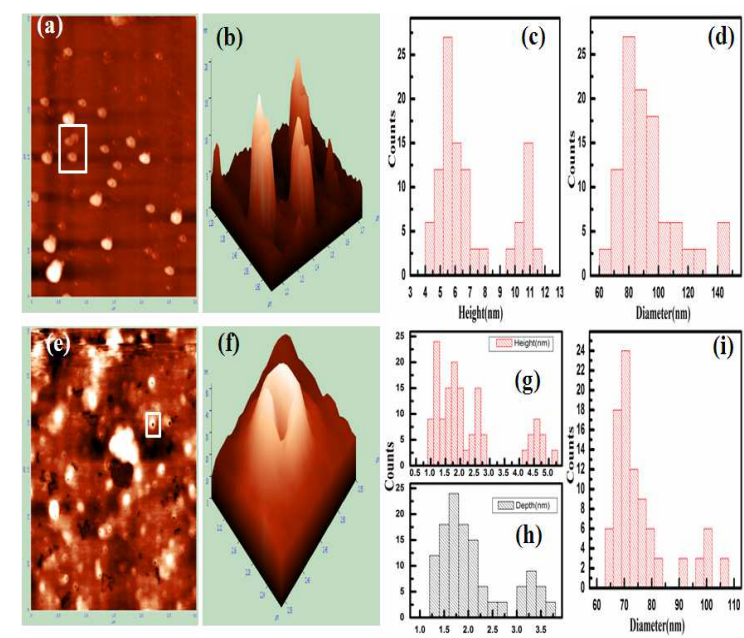

Figure 3 (a) AFM topography of PST3K PGNP incubated SLBs. (b) 3D representation of a typical barrel-like structure corresponding to the white box in panel (a). (c) Height and (d) diameter distribution of the self assembled barrel-like structures in (b). (e) AFM Topography of PST53K PGNP incubated SLBs. (f) 3D representation of a typical pore like structure corresponding to the white box in panel (e).(g) Height (h), depth and (i) diameter distribution of pore like features shown in (f).

molecules and demonstrates the versatility of these plasmonic bio-inspired membranes as potential bio-chemical sensors.

\section{Results \& discussions}

We first discuss the results of photoluminescence (PL) measurements of three typical dye molecules widely used as bio-chemical markers [31, 32, 33, 34, 35, 36]- Atto488(At)[Invitrogen LT], rhodamine(Rh)[sigma Aldrich] and Cyanine-5(CY5)[GE Healthcare LS]. The goal is to understand if the detection sensitivity of these molecules can be altered through surface plasmon based interaction of hydrophobic PGNPs embedded in suitable phospholipid bilayer membranes. It has been suggested through molecular simulations [37, 38, 39, 40, 41, 42] and some experiments [37, 19, 43, 44, 45] that hydrophobic nanoparticles of appropriate size could be localized within the hydrophobic core of the lipid membrane. However, to our knowledge, there are no studies which indicate that plasmonic resonances of lipid membrane-embedded hydrophobic nanoparticles can be utilized to obtain enhanced detection sensitivity of molecules especially those that are biologically relevant. It may be noted here that there has been some effort, recently, in using large metal nanoparticles encapsulated by lipid bilayer can act as efficient surface enhanced Raman scattering(SERS) agents for detection of molecules [46, 47, 48].

In Fig.1(a)-(f) we show PL spectra of At, Rh and CY5 in the presence of small unilamellar vesicles(SUVs) of 1,2-dimyristoyl-sn-glycero-3phosphocholine(DMPC)[Avanti Polar Lipids], with and without the PGNPs. Incubation of these SUV's with the PGNPs of two different molecular weights (3K $\& 53 \mathrm{~K}$ ) and subsequent incubation of the combined PGNP-SUV system in At \& Rh solutions of appropriate concentrations shows a clear enhancement of the PL emission intensity for identical dye concentration and incident radiation power. It seems that for similar concentration of $\mathrm{Au}$ in PGNPs the enhancement is larger for the 53K grafted PGNP compared to the $3 \mathrm{~K}$ particles. For CY5, on the other hand, presence of the PGNPS on SUVs seems to quench the PL. The observed difference in PL intensity could be related to the resonance energy transfer process between the particles and the dye molecules and hence is related to the extent of their spectral overlap [49, 3, 50, 51, 52, 53, 54] (SI Fig 1). In fact the extent of PL enhancement seems to increase with increase in spectral overlap (SI Fig. 1) from CY5 to At. At this stage we ask the question, whether the presence of vesicles is required for the observed PL intensity variation. For this purpose, we have measured the bulk PL of At, Rh and CY5 molecules in presence of PGNPs but without any SUVs. In these experiments we have incubated the PGNPs and the dye at the same concentration for 30 minutes. Except for the presence of vesicles, other experimental conditions were maintained to be similar to that described earlier, in terms of excitation power, PGNP concentration etc. Surprisingly, here we observed an opposite behavior in terms of quenching of fluorescence emission intensity in presence of PGNPs for At \& Rh while for CY5 the behavior essentially remains the 

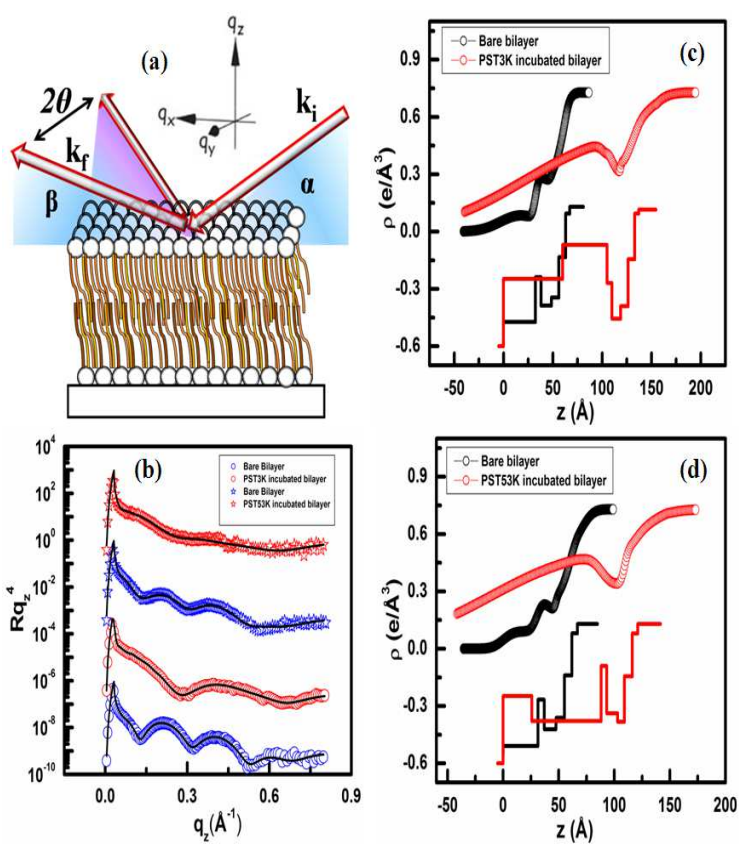

Figure 4 (a) Schematic showing the geometry of GIXS experiments. (b) Fresnel normalized XR data for SLBs before and after incubation with PST3K PGNP and PST53K PGNP. Bare SLB (O), SLB incubated with PST3K PGNPs $(\bigcirc)$, bare SLB (*r), SLB incubated with PST53K PGNPs ( 2 ). Solid line represents the corresponding best fit with the proposed model. (c)-(d) corresponding variation in electron density $(\rho)$ as a function of distance(z) (EDP) for PST3K PGNPs and PST53K PGNPs incubated SLB respectively. The profiles in solid lines in panels (c)-(d) indicate the corresponding box model representation of $\rho$ as a function of distance. The respective curves have been shifted down by (0.6) for clarity. same as in the presence of SUVs. These experiments suggests the critical role of PGNP binding to the lipid membranes of the SUVs in determining the interaction and PL from the dye molecules. It is well known that the resonance energy transfer between metal nanoparticles and the emitters is dependent on their relative separation and PL from emitters is known to be quenched at very short distances near a metal surface. The difference in PL intensity with and without SUVs is suggestive of an increase in the separation between the gold core of the PGNPs and dye (At \& Rh) molecules when the PGNPs are embedded in the lipid membrane of SUV's. It also suggests the possibilities the $\mathrm{Rh}$ (and At) is located closer to the lipid head group while CY5 might be placed within the membrane core.

To quantify exactly how the bio-mimetic lipid membranes help the PGNPs to modify the dye molecule emission enhancement, and especially to obtain a microscopic picture of the PGNP configuration on the lipid bilayers, we, further, decided to use DMPC SLBs as a biomembrane platform to repeat some of the experiments performed with DMPC SUVs. The DMPC SLBs were prepared using the well established [55, 56, 57|LangmuirBlodgett (LB) technique and transferred on glass slides (Hecht-Assistent, Germany), as described in methods and elsewhere [58], at surface pressure of 33-35 mN/m. Figure. 2(a)-(c) shows the confocal images(Leica SP5 Confocal Microscopy, Germany) of such SLBs incubated first with PGNPs at appropriate concentrations and subsequently with Rh and CY5 dye molecules. For comparison, images were also collected from pristine SLBs without being exposed to the PGNPs but incubated just in $\mathrm{Rh}$ and CY5. The images were collected at lowest laser power( $1.5 \mathrm{~mW})$ to minimize photo-toxicity of the dye molecules. A clear enhancement in brightness of the the objects (collection of dye molecules) in the respective images in presence of PGNPs with respect to the pristine SLBs shows the effect of fluorescence enhancement while exciting with the same power of Ar 488 laser line. Images with CY5 were difficult to image under similar conditions and to visualize the CY5 molecules on PGNP laden SLBs one had to increase the laser intensity considerably, indicating possible quenching of the dye fluorescence in the presence of PGNPs. To quantify the observed PL intensity variation of these dyes, we have in addition performed PL measurements with WITec alpha SNOM confocal mode 
using the green line (514 nm) of an Ar ion laser and Red line $(633 \mathrm{~nm})$ of a He-Ne laser using methodology described earlier. [59, 60, 61]. To quantify average PL enhancement/quenching, we have taken PL spectra from several places of the respective dye incubated PGNPDMPC SLBs. We observed a large enhancement in $\mathrm{Rh}$ emission, when excited with Ar 514 nm laser, in presence of PST53K PGNPs compared to the pristine SLBs, whereas enhancement is limited for PST3K nanoparticle incubated bilayer as shown in Fig. 2(d). On the other hand for CY5 dye we the PL was quenched, when excited with $633 \mathrm{~nm} \mathrm{He}-\mathrm{Ne}$ laser, in presence of PGNPs[Fig. 2(e)]. This observation also supports the qualitative trends observed for the dye molecules with SUVs.

To obtain a microscopic picture of the configuration of the PGNPs on the DMPC SLBs we have performed detailed atomic force microscopy(AFM), X-ray reflectivity (XR)and grazing incidence diffraction (GID) measurements on these samples. Figure 3 shows AFM images of DMPC SLBs transferred at surface pressure $\Pi=33 \mathrm{mN} / \mathrm{m}$ and incubated with PGNPs for a fixed concentration $(3.3 \mu \mathrm{M})$ and time(30 minutes). We observed a clear difference in the membrane mediated self assembled structures. The PGNPs capped with PST 3K forms regular barrel like structures of height 5-6 nm above the bilayer surface with a diameter $\sim 80 \mathrm{~nm}$ (much bigger than the size of a single PGNP), but there is no evidence of membrane penetration, whereas, for PGNPs capped with PST 53K we observe clear evidence of pore-like structure formation. Moreover, these structures also seem to penetrate (by $\sim 2.0 \mathrm{~nm}$ below the SLB surface) the membrane unlike those formed by the $3 \mathrm{~K}$ grafted PGNPs. Moreover, concentration dependent studies[SI Fig.2] on PST53K PGNPs identifies the approximate range $(\sim 3.3 \mu \mathrm{M})$ where it forms such kind of pore like structures, although we have not observed any such features below or above this concentration as shown in SI Fig.2(a)-(c). Thus the capping molecule plays a vital role in self assembled structure formation. Therefore AFM images seems to suggest that the self assembled configuration of PST53K PGNPs on the lipid membranes as well as their partial penetration could be responsible for observed PL enhancement in both bulk and surface PL measurements.

To obtain further insight into these aspects of PGNPSLB interactions, especially the extent of penetration of the PGNPs within the SLBs, let us turn our attention to the in-situ XR[62, 63, 64, 65, 66] and GID [67, 68, 62, 63, 69, 70] measurements performed on the DMPC SLB transferred at $\Pi=40 \mathrm{mN} / \mathrm{m}$ after they were incubated with Au-PST3K \& Au-PST53K hydrophobic PGNPs (see methods) [71]. Figure 4(b) shows XR (Fresnel normalized) data as a function of out of plane wave vector $\mathrm{q}_{z}\left(=\frac{4 \pi}{\lambda_{x}} \sin \alpha\right)$, collected on these SLBs before and after they were incubated with nanoparticles. Here $\alpha$ is the angle of incidence and $\lambda_{x}(\sim 0.49)$ is the wave length of the incident X-ray. The XR data for PST53K PGNP shows a clear drastic reduction in the total film thickness. On the other hand, the extent of changes in XR data for PST3K PGNP incubated SLB is lesser. To extract more quantitative information we have modeled the XR data using the model shown in SI fig. 5 to obtain the corresponding electron density profiles (EDP) for the respective data as shown in fig. 4(c)\&(d). Clearly, there seems to be considerably more disruption in both the top and bottom leaflet of the SLB by PST53K than by Au-PST3K PGNPs. Interestingly we observed an increment in head group electron density of the top leaflet for PST53K PGNPs incubated SLB. whereas there is a reduction in electron density for the whole tail part and the head group of the bottom leaflet. Similarly for PST3K PGNPs incubated SLBs, we observed reduction of electron density in the top leaflet only. The extracted parameters are summarized in greater details in SI Table 1 . The electron density values for both the system indicates that there is a partial penetration of PST53K PGNPs in the hydrophilic head region of the top leaflet whereas the hydrophobic chain extends up to the head group of the bottom leaflet and therefore reduces the lipid compactness and hence the electron density. On the other hand the PST3K PGNPs perturbs the top leaflet(quantified by the reduction in the electron density in the top leaflet only) but there is no significant penetration or pore formation in the DMPC SLB. From SI table 1 it is clear that the PST3K PGNPs affects only the top leaflet of the membrane and the chains partially enters in to it causing a decrease in electron density from its normal value. The reduction of thickness of the top leaflet $\sim 2.5$ represents the local perturbation and increasing chain tilting due to nanoparticle binding on the bilayer surface. The scenario is different for PST53K PGNPs as it alters the thickness and electron density profile for the top leaflet head part as well as the full hydrophobic tail region. It causes a total reduction of $\sim 1.25$ in the top 
leaflet followed by a enhancement of the electron density in both the head and tail part of the top leaflet, suggesting the partial insertion of Au core in DMPC bilayer. The particle layer in the model represents an effective composite layer of PGNPs. For PST3K PGNPs we have observed a layer of larger electron density thickness compared to PST53K PGNPs. This observed reduction of electron density satisfy the fact that in PST53K amount of grafted polymer is more than that of PST3K PGNPs. Thus, XR measurements proves our hypothesis that there is a membrane penetration by PGNPs and it is more for PST53K compared to PST3K capped particles due to a large hydrophobic polymeric shell. Moreover, this also indicates that dye molecule-Au NP core separation can be effectively enhanced with the PGNP PST53K nanoparticles for dye molecules (At and Rh) which do not seem to be favorable for penetrating into the SLB hydrophobic core and hence are more likely to be able to enhance their PL. This can help explain the observed higher detection sensitivity and PL enhancement for At and Rh in presence of, especially, PST53K PGNP. It might be noted that PGNP PST3K also penetrates partially and the hydrophobic grafted layer also adds to the separation between dye and Au NP core leading some PL enhancement. In solution, the grafted chains stretch out and are extended longer and make it possible for the dye molecule to penetrate the grafted layer (note that dye molecule sizes are considerably smaller compared to the grafted PST chains in solutions)and reach closer to the Au core leading to quenching of their PL.

To elaborate the effect of PGNP-SLB interaction, especially the role of this interaction, on SLB integrity and ordering as well possible alterations in their phase behavior and mechanical properties, we have performed GID measurements on these systems under identical conditions along with the XR measurements. In Fig. 5 we show the $3 \mathrm{D}$ reciprocal space maps of the diffracted intensities from the lipid lattice structure. The respective reciprocal lattice vectors have been defined as $\mathrm{Q}_{x y}=\frac{4 \pi}{\lambda_{x}} \sin \left(\frac{2 \theta}{2}\right)$ and $\mathrm{Q}_{z}=\frac{2 \pi}{\lambda_{x}}[\sin (\alpha)+\sin (\beta)]$ where $\alpha, \beta$ and $2 \theta$ is indicated in Fig. 4(a). In our measurements we have found two distinct peaks (representative of non-degenerate $[1,1]$ and degenerate peaks $[0,2])$ typical for the molecules arranged in a rectangular lattice symmetry [69, 68]. From the individual peak positions we have extracted the lattice pa-
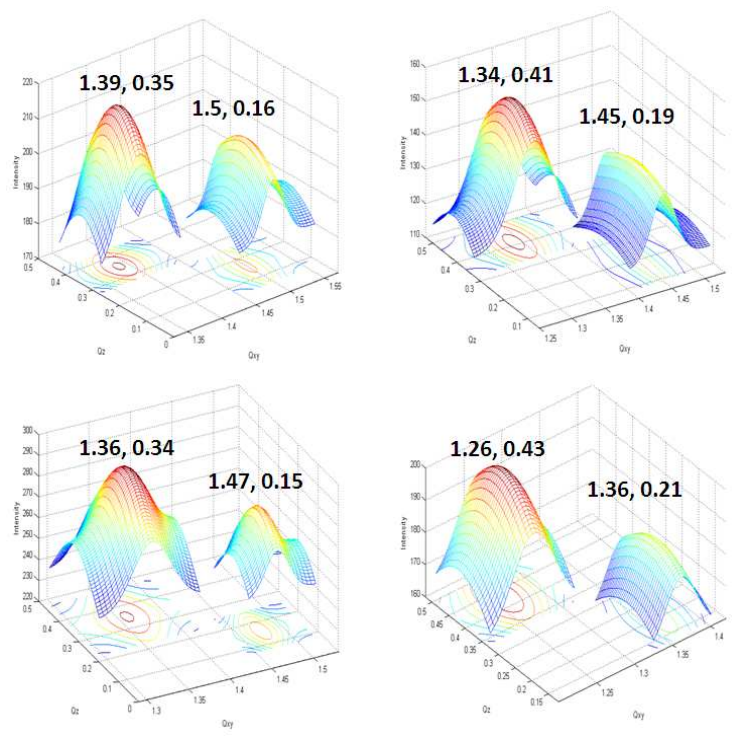

Figure 5 Reciprocal space mapping of (a) bare SLB (b) after incubation with PST3K PGNPs. (c) bare SLB (d) after incubation with PST53K PGNPs. The numbers shown in each panel identifies the exact peak position in terms of $\left(\mathrm{Q}_{x y}, \mathrm{Q}_{z}\right)$.

rameters of the rectangular lattice system tabulated in SI Table 2 using the general relations connecting interplanar spacing with Miller indices using the relation.

$$
\frac{1}{d^{2}}=h^{2} a^{*^{2}}+k^{2} b^{*^{2}}+2 h k a^{*} b^{*} \cos \left(\gamma^{*}\right),
$$

where $h$ and $k$ are the defined Miller index of a particular plane, $a^{*}$ and $b^{*}$ defines the unit cell dimension in reciprocal space and is defined in terms of real space lattice (for a rectangular lattice system) vector by

$$
a^{* 2}=\frac{1}{a^{2}}, b^{* 2}=\frac{1}{b^{2}},
$$

and $\gamma^{*}$ represents the angle for the reciprocal lattice.

From SI Table 2, it is clear that the lattice expands, without change in symmetry, when the SLBs are incubated with PGNPs. The extent of this expansion is larger for SLBs incubated with PST53K PGNPs, compared to PST3K nanoparticles under identical conditions. Apart 
from the unit cell dimensions various other parameters can be extracted by careful analysis of the GID rods. For example the in-plane lateral coherence length, $\mathrm{L}_{x y}(\mathrm{SI}$ Eqn 1), signifying the extent of in-plane ordering of lipid molecule can be extracted. Similarly, the out of plane coherence length, $\mathrm{L}_{z}$ (SI Eqn 2), represents the extent of coherence in vertical ordering can also be estimated from the data. SI Table 2 indicates the values for $\mathrm{L}_{x y}$ and $\mathrm{L}_{z}$ [58]indicating a clear reduction in both the parameters for PST3K \& PST53K PGNPs incubated SLBs as compared to the bare SLB values. PST53K PGNPs results in a $46 \%$ decrease in $\mathrm{L}_{x y}$ when compared with the bare SLB. In contrast only a $16 \%$ decrease is observed for the PST3K PGNPs-SLB system. The corresponding changes in vertical ordering, as estimated from reduction in $\mathrm{L}_{z}$, are smaller at $14.1 \%$ and $11.7 \%$ change for PST53K and Au-PST3K PGNPs when compared with the bare SLBs, respectively. These changes clearly indicate the stronger interaction with SLBs and their re-organizations caused by the PST53K PGNPs and correlates well, especially the change in $\mathrm{L}_{z}$, with the observations of reduced SLB thickness as extracted from EDP.

Besides, we have also estimated the values of $\mathrm{A}_{h}$ and area/chain $\left(\mathrm{A}_{c}\right)$ (SI Eqn. 3) perpendicular to the chain, as the packing in head and tail part of lipid molecule is different due to different degree of tilting(SI Eqn. 4). For a rectangular unit cell of dimensions $a$ and $b$, these parameters can be defined as using the the relation 67]. SI Table 2 indicates that $\mathrm{A}_{h}$ increases as a result of PGNP interaction with SLBs. While it increases by $16.6 \%$ after PST3K PGNP incubation the increase is almost by 28.2\% after PST53K PGNP incubation, again, indicating a significantly stronger interaction of the latter with the DMPC SLBs.

Further, we have also estimated the nearest neighbor tilt angle $\left(\theta_{N N}\right)$ [69] using the values of non-degenerate and degenerate peak of GID data(SI Eqn.5). Interestingly we can also observe significantly larger increase in the $\theta_{t}$ and $\theta_{N N}$ after PST53K incubation as compared to Au-PST3K PGNP incubated SLBs. Finally the order parameter, $S_{n}$, which signifies the extent of lipid ordering can also be calculated from the tilt angle information[58].

GID measurements seems to suggest that the PGNPs fluidize the SLBs through increase in $\mathrm{A}_{h}$ and the extent of fluidization is more for PST53K compared to
PST3K PGNPs. This suggests the possibility that mechanical properties of the lipid bilayer membranes could also get affected by the PGNP insertion which could also play a crucial role in the enhancement efficiency of $\mathrm{Rh}$ as discussed earlier. To measures the effect of fluidity caused by PGNPs, we have performed diffusing wave spectroscopy(DWS) [Rheolab II, LS Instruments] based micro-rheology (MR) measurements on DMPC SUVs similar to the ones used for the bulk PL measurements discussed earlier. [72]. DWS based MR measurements have been extensively used for various soft matter systems earlier [73, 74, 75, 76, 77] and provides length scale dependent rheological information over a wider frequency range as compared to conventional rheology. Both the vesicles and PGNPs were dissolved in a solvent water/tetrahydrofuran $(\mathrm{THF})=1: 1$. Experiments were carried on these samples by using $5 \mathrm{~mm}$ thick glass cubette and $687 \mathrm{~nm}$ incident laser line for scattering. In these measurements we have varied temperature from $16^{\circ} \mathrm{C}$ to $45^{\circ} \mathrm{C}$ in steps of $2^{\circ}$ to study the effect of PGNP binding on the dynamics and phase behavior of the SUVs. At each temperature, data was collected after thermally stabilizing the system. Using intensity auto correlation function $\mathrm{g}_{2}$ we have estimated mean squared displacement(MSD) and viscoelastic modulus $\left(G^{\prime} \& G^{\prime \prime}\right)$ for a particular time range using generalized Stokes-Einstein relation. Figure 6(b) shows a comparison in terms of viscoelastic modulus as a function of temperature at a fixed frequency $(\sim 100 \mathrm{rad} / \mathrm{s})$ of DMPC vesicles in presence of PST3K and PST53K PGNPs. In order to check if the presence of THF causes any disruption to the SUVs we have performed DWS measurements on them without any added PGNPs. We have not found any qualitative difference in dynamical behavior of the vesicles when they were in DI water and in presence of water / THF mixed solvent. For DMPC SUVs we observe clear evidence of a phase transition of the lipid chains at $\sim 300 \mathrm{~K}$ as expected for this system with a nonmonotonic variation of the modulus across this temperature. Interestingly, we observed a clear and significant change in mechanical properties of vesicles incubated with PST53K grafted PGNPs compared to the PST3K capped particles. The PST53K PGNPs distorted the total mechanical property represented by its lower viscous modulus which varies monotonically across the melting temperature(295-325K), whereas in presence of PST3K PGNP, the modulus shows variation similar to the pris- 
tine SUVs. Therefore the PST3K PGNPs, even if it binds on the SUVs, are unable to alter the mechanical property of the underlying lipid bilayer, whereas PST53K PGNPs clearly seems to fluidize the DMPC SUVs. These measurements, provides further evidence that PST53K PGNP has more affinity towards DMPC lipid membranes and penetrates deeper into the membrane to alter the mechanical property, to a significantly larger extent, as compared to PST3K PGNP. These results complement and corroborate the GID and XR measurements nicely and provide a microscopic understanding not only of the PGNP interaction with the lipid bilayers but also of the observed variation of PL intensity of the dye molecules.
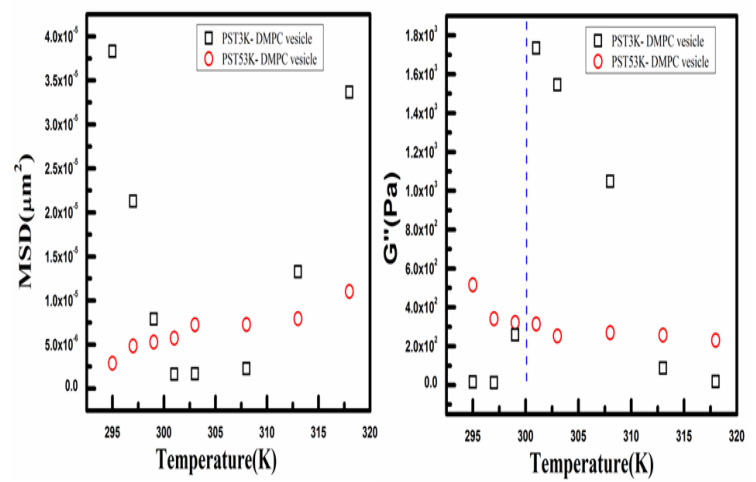

Figure 6 (a)Variation of Mean Square Displacement (at $\mathrm{t}=0.01 \mathrm{~s}$ ) with temperature for PGNP incubated DMPC lipid vesicles. (b) Dependency of the imaginary part of Visco-Elastic modulus(at $\omega \sim 100 \mathrm{rad} / \mathrm{s}$ ) for DMPC vesicle in presence of PGNPs capped with PST3K and PST53K. The blue vertical line denotes the fluid-gel transition temperature for DMPC system as reported earlier. [78]

The SUV as well as SLB based PL measurements indicate that in presence of PGNPs, emission intensity is enhanced for At and Rh whereas it quenches for CY5. The enhancement decreases from At to CY5 which can be attributed to the larger spectral overlap between the SPR of PGNPs and PL spectra of At dye, as compared to both Rh and CY5 (SI Fig 1). The effect is more prominent for PST53K PGNP compared to PST3K Surprisingly, we did not observe any enhancement in absence of lipid membranes in form of vesicles for bulk PL measure- ments. AFM images on SLB based measurements shows clear difference between the self assembled structures formed by PST3K and PST53K PGNPs. It reveals that the PST53K PGNPS penetrates inside the top leaflet of the bilayer and forms pore-like structure whereas PST3K PGNPs create surface aggregation without any signature of membrane permeation. X-ray reflectivity data analysis shows that the PST53K PGNP penetrates more into the SLBs compared to PST3K PGNPs. From EDP of the respective PGNP incubated SLBs it appears that in presence of PST3K grafted nanoparticle, the membrane perturbs locally and thus the $\rho$ of head and tail part of the top leaflet gets affected. On the other hand PST53K PGNP disturbs the full SLB. Interestingly we observed an increase of $\rho$ in the head group of the top leaflet whereas the $\rho$ has gone down for the tail and the head group of the bottom leaflet. This indicates a clear penetration of the PST53K PGNPs into the membrane and the position of hydrophilic gold core in the hydrophilic head group increased the effective $\rho$ of the top leaflet head layer in-spite of local deformation caused by the nanoparticle-membrane interaction. The height of the structures formed by the PST3K PGNPs are around 5-6 nm which is close to their total diameter (SI Table-3). Therefore such patterns formed by PST3K PGNPs are only membrane surface bound, there is no penetration. Conversely PST53K PGNPs, having comparative smaller core $\operatorname{size}(\sim 1.5 \mathrm{~nm})$ but a larger shell $(\sim 5.5 \mathrm{~nm})$, are able to penetrate in to the membrane and that is clear from the depth analysis in Fig. 2(h). The enhanced ability of the PST53K PGNPs seems to driven by the higher hydrophobicity of these particles as compared to the PST3K particles. GID measurements on PGNP-SLB systems also indicates a distortion in the lateral ordering by both the PST3K and PST53K PGNPs on DMPC SLB. A detailed analysis shows an extension of the in plane lattice dimension by around $4 \%$ for PST3K PGNP-SLB system whereas it is $\sim 7.5 \%$ for the PST53K PGNP-SLBs. We have also observed a qualitative trend in the out of plane molecular tilt angle estimation, estimating the extent of molecular distortion from its original orientation. To quantify further, we have calculated the order parameter $\left(S_{n}\right)$ for these type of systems and found the decrease in SLB is $30.69 \%$ after PST3K PGNP incubation while is value is almost $33.36 \%$ after PST53K PGNP incubation. 
Finally the DWS measurements shows that the PST53K changes the mechanical properties of the normal vesicle compared to PST3K PGNPs. As we have seen earlier also, the PST53K penetrates the membrane and make vesicles less viscoelastic, whereas the PST3K perturbs less and vesicles still shows its inherent viscoelastic nature and the phase transition across its melting temperature. The drastic reduction in the loss modulus for PST53K incubated vesicle also signifies a reduction of viscosity and hence the induced local fluidity in the lipid membrane upon binding which eventually increases continuously with temperature and destroys the DMPC lipid gel-fluid phase transition. We would like to add here that this was more a proof-of-principle experiment which demonstrated the possibility of creating such biomimetic membrane and polymer grafted nanoparticles hybrids for enhancing detection sensitivity of dyes using the plasmon resonance of the nanoparticles. More systematic study in terms of optimising the nanoparticles concentration as we have shown for dendrimer-membrane hybrids [58] or applying this to other types of plasmonic nanoparticles or polymers or even different lipid compositions would further improve the versatility of these templates.

\section{Conclusions}

In conclusion, with the combination of PL, high resolution GIXS and AFM studies we have shown probable applications of such nanoparticle embedded plasmonic biomembranes in terms for enhancing bio sensitivity using several important biomarkers. We have also provided mechanistic insight on the PGNP-lipid bilayer interaction and formation of regular patterns as well as possible molecular level reorganizations in the lipid membrane. We observe, a very regular and well defined barrel-like and pore like structure by membrane bound nanoparticles, without actually disrupting the integrity of the membranes which could affect the PL intensity enhancement for bio-compatible emitters. Such structures help in controlling the distances between the acceptors and donors and hence maximizing the emission. These results on hydrophobic nanoparticles could have potential applications in the field of bio-sensors, bio-signaling, bio-imaging, immunology, photothermal therapy as well as in target spe- cific drug/gene delivery.

\section{Acknowledgment}

We acknowledge a DST-IRHPA grant for financial support. R.B and CI acknowledges CSIR for financial support. Authors acknowledge DST and SINP for financing and facilitating the synchrotron experiments at PETRAIII under INDIA-DESY collaboration. Authors acknowledge Dr. Carsten Deiter of European XFEL GmbH for assistance during measurements at PETRA III, DESY.

\section{References}

[1] J. N. Anker, W. P. Hall, O. Lyandres, N. C. Shah, J. Zhao, R. P. V. Duyne. Nat. Mater. 2008, 7, 442.

[2] A. G. Brolo. Nat. Photonics 2012, 6(11), 709.

[3] C. Sönnichsen, B. M. Reinhard, J. Liphardt, A. P. Alivisatos. Nat. Biotechnol. 2005, 23, 741.

[4] I. H. El-Sayed, X. Huang, M. A. El-Sayed. Nano Lett. 2005, 5, 829.

[5] W. C. Law, K. T. Yong, A. Baev, P. N. Prasad. ACS Nano 2011, 5(6), 4858.

[6] V. V. Thacker, L. O. Herrmann, D. O. Sigle, T. Zhang, T. Liedl, J. J. Baumberg, U. F. Keyser. Nat. Comm. 2014, 5, 1.

[7] J. F. Li, X. D. Tian, S. B. Li, J. R. Anema, Z. L. Yang, Y. Ding, Y. F. Wu, Y. M. Zeng, Q. Z. Chen, B. Ren. Nat. Protocols 2013, 8(1), 52.

[8] X. Qian, X. H. Peng, D. O. Ansari, Q. Y. Goen, G. Z. Chen, D. M. Shin, L. Yang, A. N. Young, M. D. Wang, S. Nie. Nat. Biotechnol. 2008, 26, 83.

[9] A. F. Palonpon, J. Ando, H. Yamakoshi, K. Dodo, M. Sodeoka, S. Kawata, K. Fujita. Nat. Protocols 2013, 8, 677.

[10] P. P. Patra, R. Chikkaraddy, R. P. Tripathi, A. Dasgupta, GV. P. Kumar. Nat Comm. 2014, 5, 1. 
[11] T. Zhang, G. Lu, H. Shen, K. Shi, Y. Jiang, D. Xu, Q. Gong. Sci. Rep. 2014, 4, 1.

[12] C. Lumdee, B. Yun, P. G. Kik. ACS Photonics 2014, $1,1224$.

[13] H. Hu, H. Duan, J. K. W. Yang, Z. X. Shen. ACS Nano 2012, 6, 10147.

[14] V. Knittel, M. P. Fischer, T. D. Roo, S. Mecking, A. Leitenstorfer, D. Brida. ACS Nano 2015, 9, 894.

[15] S. J. Marrink, H. J. C. Berendsen. J. Phys. Chem. 1996, 100, 16729.

[16] V. V. Ginzburg, S. Balijepalli. Nano Lett. 2007, 7, 3716.

[17] R. Qiao, A. P. Roberts, A. S. Mount, S. J. Klaine, P. C. Ke. Nano Lett. 2007, 7, 614.

[18] A. Kessel, D. S. Bechor, T. Haliloglu, N. B. Tal. Biophys. J. 2003, 85, 3431 .

[19] M. Schulz, A. Olubummo, W. H. Binder. Soft Matt. 2012, 8, 4849 .

[20] S. Hong, A. U. Bielinska, A. Mecke, B. Keszler, J. L. Beals, X. Shi, L. Balogh, B. G. Orr, J. R. Baker, M. M. B. Holl. Bioconj. Chem. 2004, 15, 774.

[21] Y. Roiter, M. Ornatska, A. R Rammohan, J. Balakrishnan, D. R. Heine, S. Minko. Nano Lett. 2008, 8, 941.

[22] A. G. Lee. Biochem. et Biophys. Acta (BBA)Biomembranes 2003, 1612, 1.

[23] Y. Sonntag, M. Musgaard, C. Olesen, B. Schiøtt, J. V. Møller, P. Nissen, L. Thøgersen. Nat. Comm. 2011, 2, 304

[24] B. Jing, Y. Zhu. J. Am. Chem. Soc. 2011, 133, 10983.

[25] C. V. Kelly, P. R. Leroueil, B. G. Orr, M. M. B. Holl, I. Andricioaei. J. Phys. Chem. B 2008, 112, 9346.

[26] B. D. Chithrani, A. A. Ghazani, W. C. W. Chan. Nano Lett. 2006, 6, 662.
[27] M. Fosnaric, A. Iglic, S. May. Phys. Rev. E 2006 , $74,051503$.

[28] H. Lee, R. G. Larson. J. Phys. Chem. B 2006, 110, 18204.

[29] M. Fosnaric, V. K. Iglic, K. Bohinc, A. Iglic, S. May. J. Phys. Chem. B 2003, 107, 12519.

[30] N. Biswas, R. Bhattacharya, A. Saha, N. R. Jana, J. K. Basu. Phys. Chem. Chem. Phys. 2015, DOI: 10.1039/C5CP04002G.

[31] C. Commisso, R. J. Flinn, D. B. Sagi. Nat. Protocols 2014, 9, 182.

[32] C. Srinivasan, J. Lee, F. Papadimitrakopoulos, L. K. Silbart, M. Zhao, D. J. Burgess. Mol. Therapy 2006, 14, 192.

[33] L. V. Johnson, M. L. Walsh, L. B. Chen. Proc. Nat. Acad. Sci. 1980, 77, 990.

[34] H. W. Allenspach, M. Guenthert, S. Ott. Biochem. 1993, 32, 900

[35] A. Iqbal, L. Wang, K. C. Thompson, D. M. J. Lilley, D. G. Norman. Biochem. 2008, 47, 7857.

[36] C. S. Huang, S. George, M. Lu, V. Chaudhery, R. Tan, R. C. Zangar, B. T. Cunningham. Anal. Chem. 2011, 83, 1425.

[37] R. C. V. Lehn, M. Ricci, P. H. J. Silva, P. Andreozzi, J. Reguera, K. Voïtchovsky, F. Stellacci, A. A. Katz. Nat. Comm. 2014, 5, 1.

[38] S. Pogodin, M. Werner, J. U. Sommer, V. A. Baulin. ACS Nano 2012, 6, 10555.

[39] A. R. Mhashal, S. Roy. PloS One 2014, 9, 114.

[40] Y. Li, X. Chen, N. Gu. J. Phys. Chem. B 2008, 112, 16647.

[41] Y. Li, X. Li, Z. Li, H. Gao. Nanoscale 2012, 4, 3768.

[42] F. Liu, D. Wu, R. D. Kamm, K. Chen. Biochem. et Biophys. Acta (BBA)-Biomembranes 2013, 1828, 1667. 
[43] B. Jing, R. C. T. Abot, Y. Zhu. J. Phys. Chem. B 2014, 118, 13175.

[44] G. D. Bothun. J. Nanobiotechnol 2008, 6, 13.

[45] P. B. Santhosh, S. Penič, J. Genova, A. Iglič, V. K. Iglič, N. P. Ulrih. In J. Phys.: Conf. Series 2012, 398, 012034 .

[46] A. F. Stewart, A. Lee, A. Ahmed, S. Ip, E. Kumacheva, G. C. Walker. ACS nano 2014, 8, 5462.

[47] C. R. Zamecnik, A. Ahmed, C. M. Walters, R. Gordon, G. C. Walker. J. Phys. Chem. C 2013, 117, 1879.

[48] S. Ip, C. M. MacLaughlin, N. Gunari, G. C. Walker. Langmuir 2011, 27, 7024.

[49] E. Dulkeith, AC. Morteani, T. Niedereichholz, T. A. Klar, J. Feldmann, S. A. Levi, FCJM. V. Veggel, DN. Reinhoudt, M. Möller, DI. Gittins. Phys. Rev. Lett. 2002, 89, 203002.

[50] G. P. Acuna, M. Bucher, I. H. Stein, C. Steinhauer, A. Kuzyk, P. Holzmeister, R. Schreiber, A. Moroz, F. D. Stefani, T. Liedl. ACS Nano 2012, 6, 3189.

[51] F. Gao, P. Cui, X. Chen, Q. Ye, M. Li, L. Wang. Analyst 2011, 136, 3973.

[52] S. T. Kochuveedu, T. Son, Y. Lee, M. Lee, D. Kim, D. H. Kim. Sci. Rep. 2014, 4, 1.

[53] D. Ghosh, N. Chattopadhyay. Opt. Photon. J. 2013, $3,18$.

[54] N. T. Chen, S. H. Cheng, C. P. Liu, J. S. Souris, C. T. Chen, C. Y. Mou, L. W. Lo. Int. J. Mol. Sci. 2012, 13, 16598.

[55] J. Liu, J. C. Conboy. Langmuir 2005, 21, 9091.

[56] J. Y. Wong, J. Majewski, M. Seitz, C. K. Park, J. N. Israelachvili, G. S. Smith. Biophys. J. 1999, 77, 1445 .

[57] J. Liu,J. C. Conboy. J. Am. Chem. Soc. 2004, 126, 8894.
[58] R. Bhattacharya, S. Kanchi, C. Roobala, A. Lakshminarayanan, O. H. Seeck, P. K. Maiti, KG. Ayappa, N. Jayaraman, J. K. Basu. Soft Matt. 2014, 10, 7577.

[59] M. Haridas, L. N. Tripathi, J. K. Basu. Appl. Phys. Lett. 2011, 98, 063305.

[60] L. N. Tripathi, M. Praveena, P. Valson, J. K. Basu. Appl. Phys. Lett. 2014, 105, 163106.

[61] C. Indukuri, A. Mukherjee, J. K. Basu. Appl. Phys. Lett. 2015, 106, 131111.

[62] E. B. Watkins, C. E. Miller, J. Majewski, T. L. Kuhl. Proc. .Nat. Acad. Sci. 2011, 108, 6975.

[63] E. B. Watkins, C. E. Miller, D. J. Mulder, T. L. Kuhl, J. Majewski. Phys. Rev. Lett. 2009, 102, 238101.

[64] O. H. Seeck, H. Kim, D. R. Lee, D. Shu, I. D. Kaendler, J. K. Basu, S. K. Sinha. Europhys. Lett. 2002, 60, 376 .

[65] C. M. DeCaro, J. D. Berry, L. B. Lurio, Y. Ma, G. Chen, S. K. Sinha, L. Tayebi, A. N. Parikh, Z. Jiang, A. R. Sandy. Phys. Rev. E 2011, 84, 041914.

[66] S. Chandran, J. K. Basu, M. K. Mukhopadhyay. J. Chem. Phys. 2013, 138, 014902.

[67] W. J. Sun, R. M. Suter, M. A. Knewtson, C. R. Worthington, S. T. Nagle, R. Zhang, J. F. Nagle. Phys. Rev. E 1994, 49, 4665.

[68] J. A. Nielsen, D. Jacquemain, K. Kjaer, F. Leveiller, M. Lahav, L. Leiserowitz. Phys. Rep. 1994, 246, 251.

[69] V. M. Kaganer, H. Möhwald, P. Dutta. Rev. Mod. Phys. 1999, 71, 779.

[70] S. K. Ghosh, S. Castorph, O. Konovalov, T. Salditt, R. Jahn, M. Holt. Biophys. J. 2012, 102, 1394.

[71] O. H. Seeck, C. Deiter, K. Pflaum, F. Bertam, A. Beerlink, H. Franz, J. Horbach, H. S. Schrepping, B. M. Murphy, M. Greve. J. Synch. Radiation 2011, $19,30$.

[72] D. A. Weitz, J. X. Zhu, D. J. Durian, H. U. Gang, and DJ Pine. Physica Scripta 1993, T49B, 610. 
[73] D. J. Pine, D. A. Weitz, P. M. Chaikin, E. Herbolzheimer. Phys. Rev. Lett. 1988, 60, 1134.

[74] G. Maret, P. E. Wolf. Zeitschrift für Physik B Cond. Mater. 1987, 65, 409.

[75] N. Menon, D. J. Durian. Science 1997, 275, 1920.

[76] B. R. Dasgupta, D. A. Weitz. Phys. Rev. E 2005, 71, 021504 .

[77] J. L. Harden, V. Viasnoff. Current Opinion in Coll. \& Int. Sci. 2001, 6, 438.

[78] C. W. Harland, M. J. Bradley, R. Parthasarathy. Proc. Nat. Acad. Sci. 2010, 107, 19146. 